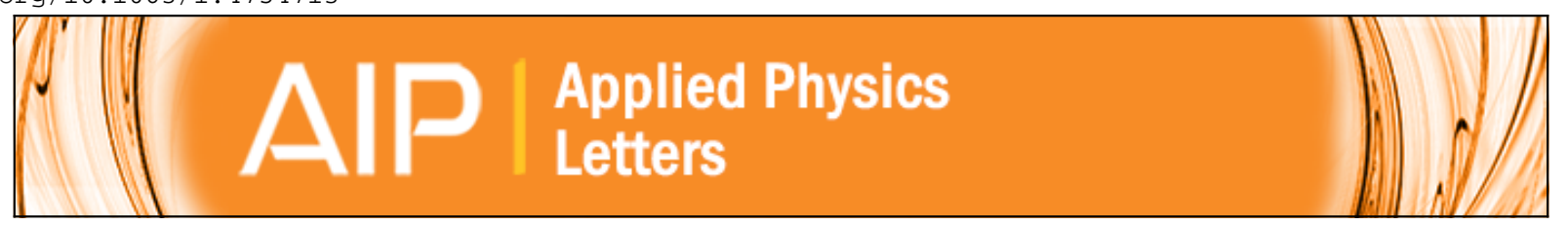

Self-assembled NaNbO3-Nb2O5 (ferroelectric-semiconductor) heterostructures grown on LaAIO3 substrates

Zhiguang Wang, Yanxi Li, Bo Chen, Ravindranath Viswan, Jie-Fang Li, and D. Viehland

Citation: Applied Physics Letters 101, 132902 (2012); doi: 10.1063/1.4754713

View online: http://dx.doi.org/10.1063/1.4754713

View Table of Contents: http://scitation.aip.org/content/aip/journal/apl/101/13?ver=pdfcov

Published by the AIP Publishing

AAP Re-register for Table of Content Alerts 


\title{
Self-assembled $\mathrm{NaNbO}_{3}-\mathrm{Nb}_{2} \mathrm{O}_{5}$ (ferroelectric-semiconductor) heterostructures grown on $\mathrm{LaAlO}_{3}$ substrates
}

\author{
Zhiguang Wang, ${ }^{\text {a) }}$ Yanxi Li, Bo Chen, Ravindranath Viswan, Jie-Fang Li, and D. Viehland \\ Department of Materials Science and Engineering, Virginia Tech, 306 Holden Hall, Blacksburg, \\ Virginia 24061, USA
}

(Received 4 July 2012; accepted 11 September 2012; published online 24 September 2012)

\begin{abstract}
We deposited $\mathrm{NaNbO}_{3}$ (NNO)- $\mathrm{Nb}_{2} \mathrm{O}_{5}$ (NO) self-assembled heterostructures on $\mathrm{LaAlO}_{3}$ (LAO) to form ferroelectric-semiconductor vertically integrated nanostructures. The NNO component formed as nanorods embedded in a NO matrix. X-ray diffraction confirmed epitaxial growth of both NNO and NO phases. Phase distribution was detected by scanning electron microscopy. The NNO/NO volume ratio was strongly dependent on the deposition temperature due to the volatility of sodium. Piezoelectric force microscopy revealed a good piezoelectric response in the NNO component with a piezoelectric coefficient of $\mathrm{D}_{33} \approx 12 \mathrm{pm} / \mathrm{V}$, with $\mathrm{SrRuO}_{3}$ (SRO) acting as bottom electrode. The current-voltage characterization of NNO-NO/SRO-LAO showed a typical diode rectifying behavior. (C) 2012 American Institute of Physics. [http://dx.doi.org/10.1063/1.4754713]
\end{abstract}

Two-phase hetero-nanostructures are an important new class of materials which hold great promise for multifunctional device applications due to a coupling of the different properties from each component phase. ${ }^{1-3}$ First, heteronanostructure systems could offer significant advantages over single components systems. For example, $\mathrm{BiFeO}_{3}$ (BFO) $-\mathrm{Sm}_{2} \mathrm{O}_{3}(\mathrm{SmO})$ vertically aligned thin film shows much smaller dielectric loss compared with that of BFO and $\mathrm{SmO}$ ones due to elastic strain in the perpendicular interface. ${ }^{4}$ In addition, $\mathrm{BaTiO}_{3}$ (BTO)-SmO self-assembled thin films have an enhanced phase stability due to the strain from the SmO nanopillars embedded in the BTO matrix. ${ }^{5}$ Second, these two-phase hetero-nanostructures offer the possibility of achieving multifunctionality. For example, the coupling between ferroelectric and ferromagnetic properties in $\mathrm{BaTiO}_{3}-\mathrm{CoFe}_{2} \mathrm{O}_{4}$ allows exchange between electric and magnetic fluxes. Such magnetoelectric coupling has potential importance for magnetic sensors and electric field controlled magnetic memory devices. ${ }^{6,7}$ The magnitude of the coupling between the two component phases is proportional to the interfacial area between them, where the elastic strain acts as a coupling mechanism. Vertically aligned heterostructures have much larger interfacial area compared to traditional multilayer structures, and in addition suffer less substrate clamping effect. Thus, we expect much larger coupling effects and higher multifunctionality for vertically aligned nanostructures.

Here, we focused on the growth of ferroelectric/semiconductor nano-heterostructures due to their possible applications in multifunctional devices. ${ }^{8,9}$ The $\mathrm{NaNbO}_{3}$ (NNO) phase exhibits good ferroelectric properties in thin film form, and $\mathrm{Nb}_{2} \mathrm{O}_{5}$ (NO) is a widely used $\mathrm{n}$ type semiconductor. The NNO-NO composite structure has been reported to have a phase configuration of NNO nanoplates embedded with NO nanotubes. ${ }^{10}$ However, this complex composite structure occurs only in powder form, and is thus difficult to be integrated on a substrate. To overcome these limitations, we used pulsed laser deposition for the growth of NNO-NO

${ }^{a)}$ E-mail: zgwang@vt.edu. layers which spontaneously phase separated into vertically aligned heterostructural layers on the substrate. Such layers offer an ease of characterization, and the potential to be integrated with semiconductor technologies.

We designed an oxide target with a composition ratio of 66 at. $\%$ NNO-34 at. \%NO. The ablation target was made by mixing and grinding $\mathrm{NaCO}_{3}$ and $\mathrm{Nb}_{2} \mathrm{O}_{5}$ powders in the relevant ratios. The targets were pressed and sintered for $4 \mathrm{~h}$ at $1000{ }^{\circ} \mathrm{C}$. During sintering the target was contained within a closed crucible to reduce the evaporation of sodium. NNO and NO targets were prepared in the same way with stoichiometric ratio for comparison of the crystal and electric properties. Since conditions for growth of NNO on $\mathrm{LaAlO}_{3}$ (LAO) are well established, ${ }^{11}$ LAO was chosen as the substrate. $\mathrm{SrRuO}_{3}$ (SRO) bottom electrode with a thickness of $50 \mathrm{~nm}$ was first deposited on (001) oriented LAO at $650{ }^{\circ} \mathrm{C}$, using $\mathrm{KrF}$ excimer laser $(\lambda=248 \mathrm{~nm})$. Then, we deposited NNO-NO epitaxial thin films at different deposition temperatures of $650{ }^{\circ} \mathrm{C}, 700^{\circ} \mathrm{C}$, and $750{ }^{\circ} \mathrm{C}$. The laser was focused to a spot size of about $2 \mathrm{~mm}^{2}$, and was incident on the surface of the target using an energy density of $2 \mathrm{~J} \cdot \mathrm{cm}^{2}$. The distance between the substrate and the target was $6 \mathrm{~cm}$, and the base vacuum of the chamber was $10^{-6}$ Torr. During film deposition, the oxygen pressure was kept at 75 mTorr. The crystal structure of the films was determined using a Philips $X$ 'pert high resolution $\mathrm{X}$-ray diffractometer configured for single crystal measurements. The morphology of the thin films was characterized by scanning electron microscopy (SEM), using a LEO (Zeiss) 1550. Piezoelectric force microscopy (PFM) in a contact mode was used to image the ferroelectric regions and measure their piezoelectric coefficients (Veeco SPI 3100).

Figure 1(a) shows a schematic of the phase distribution of a NNO-NO self-assembled structure on LAO. The NNO component formed as nanorods embedded in a NO matrix. NNO has a complex sequence of temperature-induced phase transitions and the thin film form often shows different phase configurations compared with bulk materials. Here, the NNO component has a pseudocubic perovskite structure with a crystal lattice parameter $\mathrm{a}=3.91 \AA$, and it has a modest 
(a)

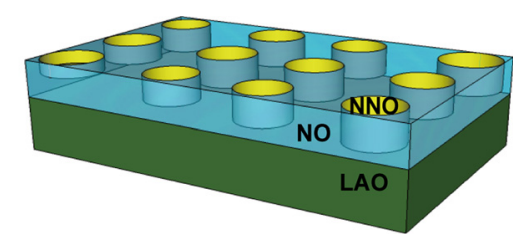

(b)

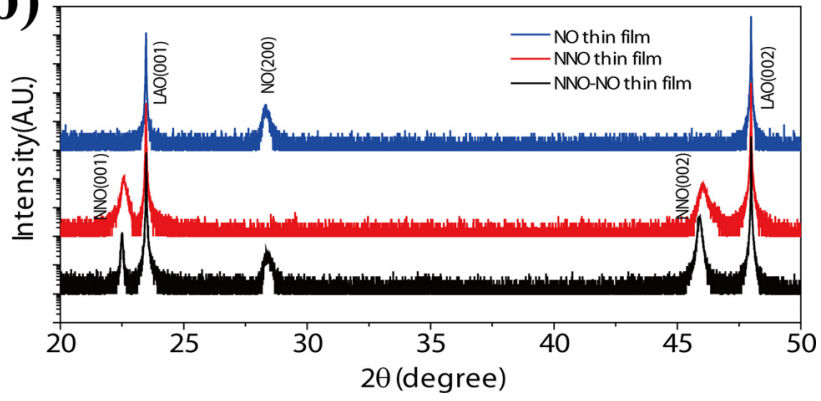

FIG. 1. (a) Schematic of a NNO-NO self-assembled thin film on a LAO substrate; (b) XRD line scans comparing the NNO-NO composite thin films with pure $\mathrm{NNO}$ and $\mathrm{NO}$ ones on LAO substrates.

lattice mismatch with the cubic LAO substrate $(a=3.82 \AA)$. Therefore, growth of NNO films on a LAO substrate follows a layer-on-layer growth mechanism. The NO phase is orthorhombic with crystal lattice parameters of $\left(\mathrm{a}_{0}, \mathrm{~b}_{0}, \mathrm{c}_{0}\right)$ $=(6.168 \AA, \quad 29.312 \AA, \quad 3.936 \AA)$, where $\mathrm{b}_{0}=8 \times 3.664 .{ }^{10}$ Thus, the NO phase can grow on LAO substrates with the following lattice heterostructural configurations: (001)NO $\|(100) \mathrm{LAO}$ and (010)NO $\|(010) \mathrm{LAO}$ in the in-plane direction, and (100)NO $\|(001) \mathrm{LAO}$ in the out-of-plane direction. Evidence of such a growth condition for NO on LAO was found by XRD line scans, which exhibited a diffraction peak near $2 \theta=28.2^{\circ}$ corresponding to the (200) plane of the NO thin film. Figure 1(b) compares the XRD line scans for NNO, NO, and two phase NNO-NO epitaxial thin films on LAO. The top (black) curve is the line scan for a pure NNO layer on LAO. Two sharp peaks located at $2 \theta=22.7^{\circ}$ and $46.38^{\circ}$ were apparent near that of LAO substrate peaks, which correspond to the (100) and (200) peaks of NNO, respectively. The middle (red) curve is the line scan for a pure $\mathrm{NO}$ layer. One sharp diffraction peak was observed at $2 \theta=28.2^{\circ}$ corresponding to the (200) peak of NO. The bottom (blue) profile is the XRD line scan for the NNO-NO composite thin film. Diffraction peaks from both the NNO and NO component phases can be seen. Clearly, NNO and NO form spontaneously separated phases when grown on LAO.

Figure 2 shows SEM images of NNO-NO heterostructures grown at different temperatures. Sodium suffers from evaporation during high-temperature deposition. Thus, thin films grown at different temperatures had different NNO/NO ratio. We studied the three different deposition temperatures of $650{ }^{\circ} \mathrm{C}, 700^{\circ} \mathrm{C}$, and $750{ }^{\circ} \mathrm{C}$. Parts (a), (b), and (c) of this figure show the morphological evolution as the deposition temperature was increased. It is obvious that the thin films deposited at the lower temperatures contained a considerably higher number of NNO nanorods, whereas the ones prepared at higher temperature had fewer and more isolated NNO nanorods presumably due to volatilization of sodium. Elemental analysis showed that the $\mathrm{NNO} / \mathrm{NO}$ volume ratio changed from 2:1 to $1: 2$ as the deposition temperature was increased from $650^{\circ} \mathrm{C}$ to $750^{\circ} \mathrm{C}$ as shown in Fig. 2(e): confirming this sodium volatilization. In addition, we determined the average size of the NNO nanorods by analysis of the SEM images. Figure 2(f) shows that the average diameter of the nanorods varied by about $10 \mathrm{~nm}$ between the various deposition temperatures. Zheng et al. ${ }^{12}$ have previously used diffusion rate versus temperature relationships to analyze the diameter change in self-assembled thin films. However, in our NNO-NO thin films, the diameter change was not monotonous, and the thin films probably had larger diameters due to abundant $\mathrm{Na}$ elements at lower deposition temperatures $\left(\mathrm{D}_{650^{\circ} \mathrm{C}}>\mathrm{D}_{700{ }^{\circ} \mathrm{C}}>\mathrm{D}_{700^{\circ} \mathrm{C}}\right)$. Figure $2(\mathrm{~d})$ shows the crosssectional SEM of the NNO-NO thin films deposited at $700^{\circ} \mathrm{C}$. It is obvious that NNO nanorods stand vertically on the substrate with uniform diameter of about $90 \mathrm{~nm}$.

Figure 3 shows PFM images and PFM- $\mathrm{D}_{33}$ measurements for a NNO-NO composite thin film deposited at $700^{\circ} \mathrm{C}$. Part (a) shows the topography of the thin film, where NNO nanorods stand out from a relative smoother NO matrix. Line profile of the topography was shown in Fig. 3(b). The altitude difference between nanorods and the matrix area is around $50 \mathrm{~nm}$. Part (c) shows the PFM response of the NNO-NO thin film. NNO nanorods show much stronger piezoelectric response than the NO matrix, resulting clear color contrast in the image. Specific piezoelectric coefficient was measured by applying an AC voltage of $3000 \mathrm{mV}$ through the conducting PFM tip. NNO-NO phase distribution could be determined by PFM image, then we could move the tip to specific positions to measure the piezoelectric response of NNO and NO, respectively. The NNO phase was piezoelectric, whereas the NO one was semiconducting. Part (d) shows well-defined $\mathrm{D}_{33}$ butterfly shape for NNO component with a piezoelectric coefficient of $\mathrm{D}_{33}=12 \mathrm{pm} / \mathrm{V}$. On the contrary, no obvious piezoelectric response could be detected in the NO matrix area.

Figure 4 shows rectifying behavior of the NNO-NO thin films with SRO as bottom electrode. Part (a) shows the modified Sawyer Tower circuit to measure the rectified conducting property of the NNO-NO/SRO heterostructure. Au square pattern with side of $50 \mu \mathrm{m}$ was prepared by sputtering, acting as top electrode. Reference capacitor $\mathrm{C}_{\mathrm{r}}=10 \mathrm{nf}$ was used to collect the charge through the sample. A triangular wave $\mathrm{V}_{\mathrm{s}}$ was introduced from signal generator. Part (b) shows the voltage on $\mathrm{C}_{\mathrm{r}}$ as a function of the triangular signal with a frequency of $1 \mathrm{~Hz}$. A diode rectifying effect is obvious: the NNO-NO/SRO heterostructure allows electric charges to flow in one direction, but not in the opposite direction, thus we could only detect triangular response $V_{c}$ on the reference capacitor when $\mathrm{V}_{\mathrm{s}}$ was positive. Part (c) shows the current-voltage (I-V) characterization of the $\mathrm{Au} /$ NNO-NO/SRO under a DC bias voltage. A turn-on voltage of $4 \mathrm{~V}$ and a forward current of $110 \mu \mathrm{A}$ were observed at $6 \mathrm{~V}$. $\mathrm{Au}$ has a work function of $5.1 \mathrm{eV}$, while that of $\mathrm{n}$ type semiconductor NO is $3.4 \mathrm{eV} .{ }^{13}$ Thus, electron has to overcome a Schottky barrier in order to move from NO to Au. In contrast to the disordered interface between Au and NO, SRO is a good heteroepitaxial metallic oxide for its eligible lattice mismatch with NNO-NO thin film. Although SRO has a larger work function $(5.2 \mathrm{eV})$ than $\mathrm{NO}^{14,15}$ the diffusion between SRO and NNO-NO prevents formation of a 

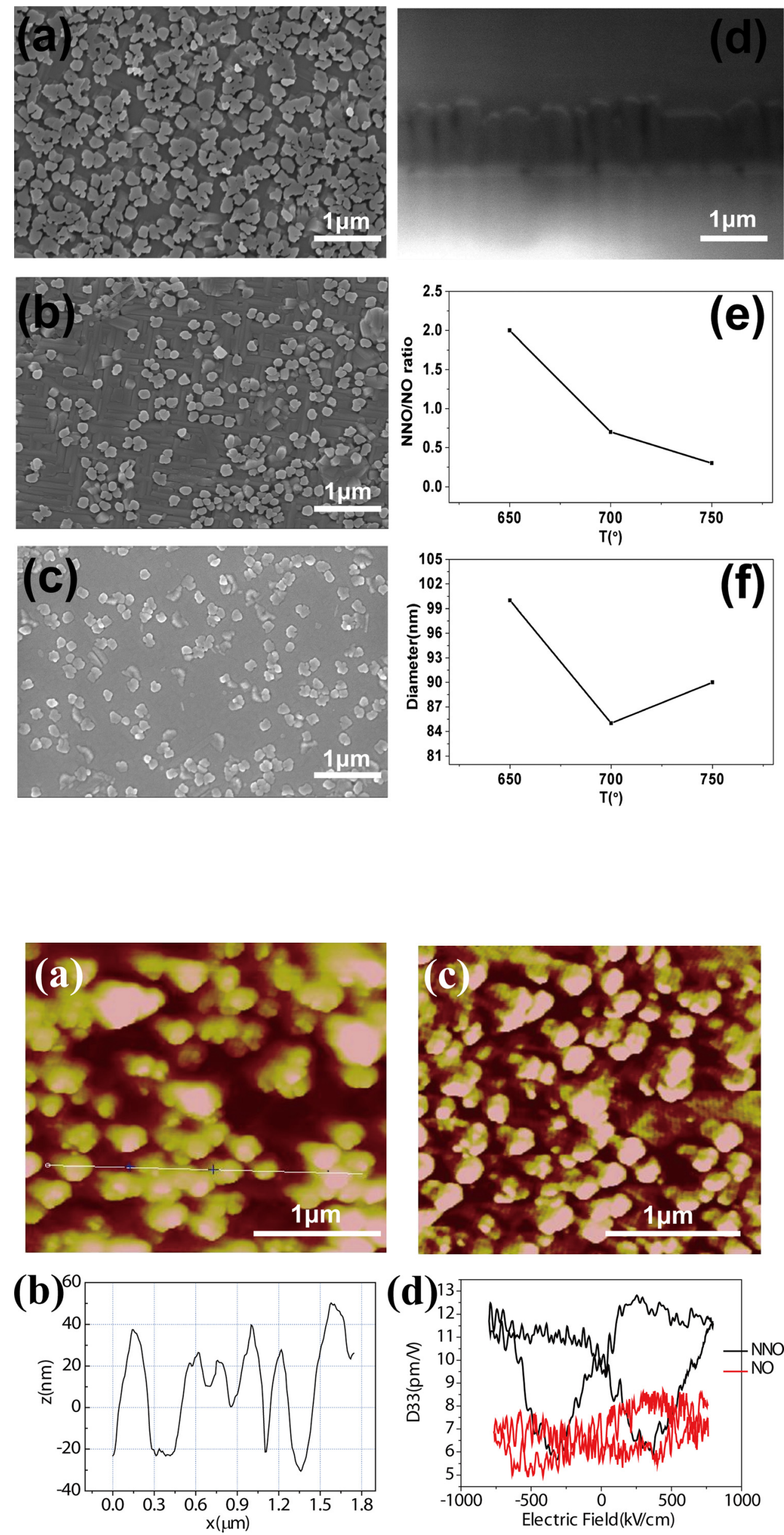

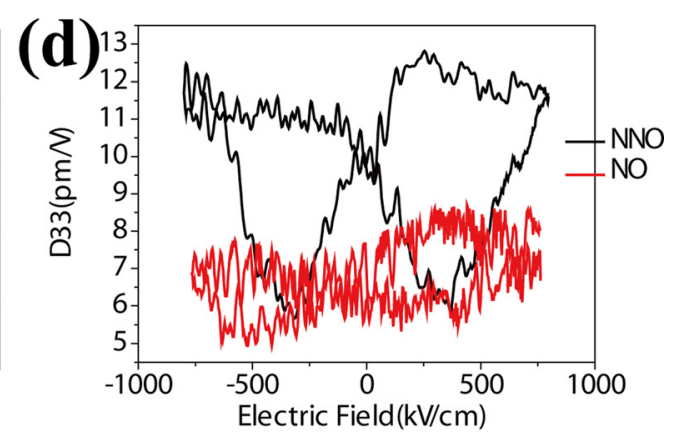

FIG. 2. (a)-(c) Top-view SEM images of NNO-NO thin films grown at different temperatures; (d) cross-sectional SEM of NNO-NO deposited at $700^{\circ} \mathrm{C}$; (e) $\mathrm{NNO} / \mathrm{NO}$ volume ratio as a function of deposition temperature; and (f) diameter of the NNO nanorods as a function of deposition temperature.
FIG. 3. (a) Topographic AFM image of NNO-NO a self-assemble thin film (scale-bar: $30 \mathrm{~nm}$ ); (b) line profile of the height in selected area in (a); (c) PFM image of NNO-NO thin films taken from the same area, showing distinct piezoelectric responses in the $\mathrm{NNO}$ and $\mathrm{NO}$ areas; and (d) comparison of $\mathrm{D}_{33}$ in $\mathrm{NNO}$ and $\mathrm{NO}$ component regions. 

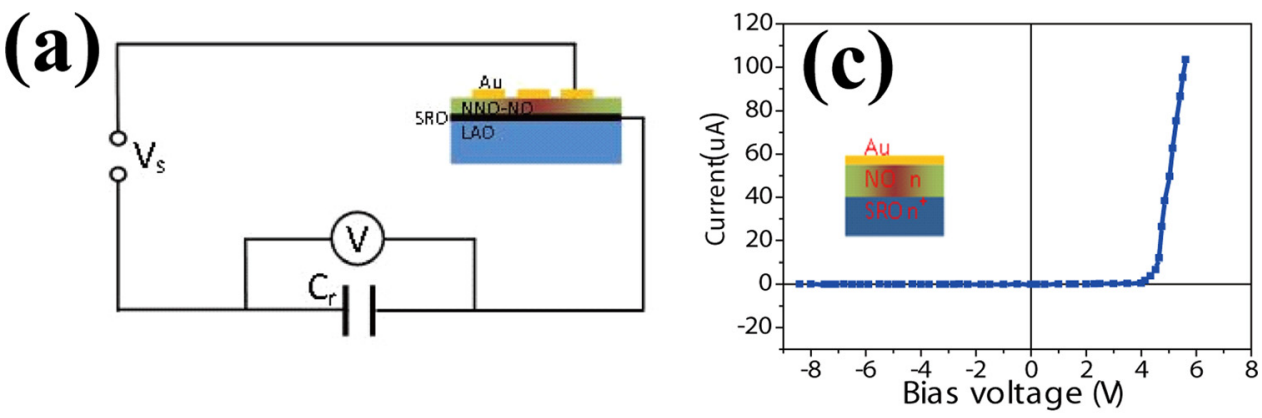

(b)

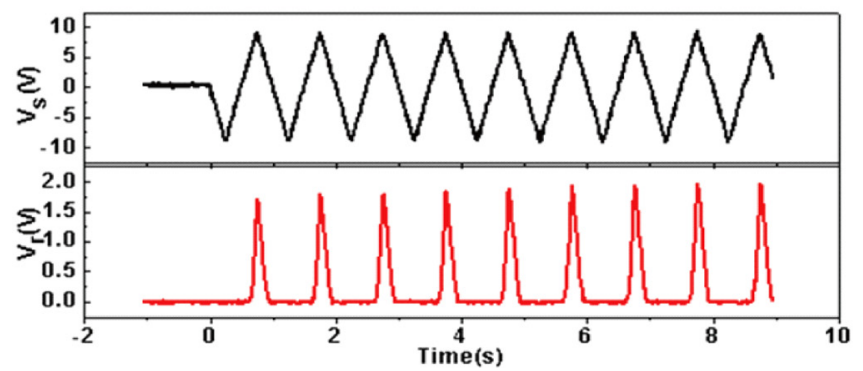

FIG. 4. (a) Modified Sawer-Tower measurement circuit; (b) triangular wave signal and the corresponding response on the reference capacitor; and (c) currentvoltage measurement of $\mathrm{Au} / \mathrm{NNO}-\mathrm{NO} /$ SRO heterostructures.
Schottky connection. SRO is a n type connector based on Hall measurement, ${ }^{16}$ so the NO/SRO could form a $n-n+$ heterojunction which has a built-in electric field with direction from NO to SRO. In this way, the NO/SRO heterojunction only allows electron to move from SRO to NO when external electric field is larger than the $n-n^{+}$barrier. The turn-on voltage is the summation of the Au/NO Schottky barrier and the $\mathrm{NO} / \mathrm{SRO} \mathrm{n}-\mathrm{n}^{+}$barrier.

In summary, we have deposited NNO-NO (piezoelectricsemiconductor) self-assembled heterostructures on LAO single crystal substrates with a controllable NNO nanorod dispersion density. The NNO nanorods had good piezoelectric properties of $\mathrm{D}_{33}=12 \mathrm{pm} / \mathrm{V}$, which were measured by a PFM characterization. I-V measurement showed a good rectifying behavior due to the formation of $\mathrm{Au} / \mathrm{NO}$ Schottky barrier and NO/SRO n- ${ }^{+}$heterojunction barrier. Our results show that NNO-NO self-assembled epitaxial layers have the dual properties of both their piezoelectric and semiconductor component phases, and thus show potential for applications in multifunctional devices.

We would like to gratefully acknowledge financial support from the U.S. Department of Energy under Contract No. DE-AC02-98CH10886 and the Office of the Air-Force Office of Scientific Research under Grant No. FA9550-09-10552 .
${ }^{1}$ H. Zheng, J. Wang, S. E. Lofland, Z. Ma, L. Mohaddes-Ardabili, T. Zhao, L. Salamanca-Riba, S. R. Shinde, S. B. Ogale, F. Bai, D. Viehland, Y. Jia, D. G. Schlom, M. Wutting, A. Roytburd, and R. Ramesh, Science 303, 661 (2004).

${ }^{2}$ F. Zavaliche, T. Zhao, H. Zheng, F. Straub, M. P. Cruz, P.-L. Yang, D. Hao, and R. Ramesh, Nano Lett. 7, 1586 (2007).

${ }^{3}$ Z. Wang, Y. Yang, R. Viswan, J. Li, and D. Viehland, Appl. Phys. Lett. 99, 043110 (2011).

${ }^{4}$ J. L. MacManus-Driscoll, P. Zerrer, H. Wang, H. Yang, J. Yoon, A. Fouchet, R. Yu, M. G. Blamire, and Q. Jia, Nature Mater. 7, 314 (2008).

${ }^{5}$ S. A. Harrington, J. Zhai, S. Denev, V. Gopalan, H. Wang, Z. Bi, S. Redfern, S.-H. Baek, C. W. Bark, C.-B. Eom, Q. Jia, M. E. Vickers, and J. L. MacManus-Driscoll, Nat. Nanotechnol. 6, 491(2011).

${ }^{6}$ Z. Wang, R. Viswan, B. Hu, J.-F. Li, V. G. Harris, and D. Viehland, J. Appl. Phys. 111, 034108 (2012).

${ }^{7}$ L. Yan, Z. P. Xing, Z. G. Wang, T. Wang, G. Y. Lie, J. F. Li, and D. Viehland, Appl. Phys. Lett. 94, 192902 (2009).

${ }^{8}$ M. Alexe, G. Kastner, D. Hesse, and U. Gosele, Appl. Phys. Lett. 70, 3416 (1997).

${ }^{9}$ T. Mokari, C. G. Sztrum, A. Salant, E. Rabani, and U. Banin, Nature Mater. 4, 855 (2005).

${ }^{10}$ C. Yan, L. Nikolova, A. Dadv, C. Harnagea, A. Sarkissian, D. F. Perepichka, D. Xue, and F. Rosei, Adv. Mater. 22, 1741 (2010).

${ }^{11}$ M. Tyunina and J. Levoska, Appl. Phys. Lett. 95, 102903 (2009).

${ }^{12}$ H. Zheng, F. Straub, Q. Zhan, P.-L. Yang, W.-K. Hsieh, F. Zavaliche, Y.-H. Chu, U. Dahmen, and R. Ramesh, Adv. Mater. 18, 2747 (2006).

${ }^{13}$ J. Sikula, J. Hlavka, V. Sedlakova, L. Grmela, P. Hoeschl, T. Zednicek, and Z. Sita, 24th Annual Capacitor and Resistor Technology Symposium, 141 (2004).

${ }^{14}$ Y. Hikita, Y. Kozuka, T. Susaki, H. Takagi, and H. Y. Hwang, Appl. Phys. Lett. 90, 143507 (2007).

${ }^{15}$ X. Fang and T. Kobayashi, Appl. Phys. A 69, S587 (1999).

${ }^{16}$ C. H. Ahn, R. H. Hammond, T. H. Geballe, M. R. Beasley, J.-M. Triscone, M. Decroux, Ø. Fischer, L. Antognazza, and K. Char, Appl. Phys. Lett. 70, 206 (1997). 